Ann. Zootech., I970, 19 (4), 4I3-422.

\title{
EVOLUTION DE LA DIGESTIBILITÉ ET DE LA QUANTITÉ INGEREE DES PLANTES ENTIÈRES D'AVOINE, DE BLE ET D'ORGE ENTRE LA FLORAISON ET LA MATURATION DU GRAIN
}

\author{
C. DEMARQUILLY \\ avec la collaboration technique de Jacqueline Aubry, \\ J.-M. Boissau et H. Bousguet \\ Station de Recherches sur l'Élevage des Ruminants, \\ Centre de Recherches de Clermont-Ferrand, \\ 6.3 - Saint-Genès-Champanelle \\ Institut national de la Recherche agronomique
}

\section{RÉSUMÉ}

La digestibilité et la quantité ingérée des plantes entières d'avoine, d'orge et de blé ont été mesurées sur des moutons, entre le stade " début épiaison " ou "floraison " et la fin du stade "pâteux ".

La participation relative des divers organes à la matière sèche de la plante évolue régulièrement jusqu'au stade pâteux (fig. 1). La teneur en matières azotées et en cellulose brute diminue au cours de la maturité, alors que la teneur en amidon augmente (tabl. I).

La digestibilité de la matière organique diminue rapidement jusqu'au début de la formation du grain où elle est de l'ordre de $6 \mathrm{I}-62$ p. I oo chez les trois céréales. Elle reste alors pratiquement constante jusqu'au stade pâteux chez l'orge et le blé ou réaugmente un peu jusqu'au stade laiteux avant de se stabiliser. En revanche, elle continue à décroître très lentement chez l'avoine (fig. 2, tabl. I). Elle est faiblement liée à la teneur en matières azotées $(r=0,733, n=27)$ et surtout à la teneur en cellulose brute $(r=0,395, n=26)$ (fig. 3).

La quantité de matière sèche ingérée diminue rapidement jusqu'au début de la formation du grain où elle est de l'ordre de $45 \mathrm{~g} / \mathrm{kg} \mathrm{P}, 75$. Elle augmente alors légèrement de to à $15 \mathrm{p}$. Ioo jusqu'au stade laiteux chez le blé et l'orge et jusqu'au stade pâteux chez l'avoine pour diminuer rapidement ensuite (fig. I).

Les quantités de matière sèche récoltées à l'hectare sont élevées et augmentent rapidement à partir de la floraison. Elles sont maximum dès le début du stade pâteux (tabl. 2).

Le stade optimum de récolte pour l'ensilage se situe au stade laiteux-pâteux, les céréales ayant alors une teneur en matière sèche comprise entre 35 et $4^{\circ} \mathrm{p}$. Ioo.

\section{INTRODUC'TION}

L'utilisation de la plante de céréale n'est pas nouvelle mais elle se faisait jusqu'à ces dernières années à un stade jeune, soit pour le pâturage au début du printemps (seigle, orge) soit pour l'ensilage (vesce-avoine). Aussi les auteurs qui comme NEHRING et BEYER (I965 et I966) ont étudié la digestibilité et la valeur nutritive du seigle et de l'avoine sur pied, l'ont fait entre la montaison et la floraison; ils ont 
montré qu'entre ces stades la digestibilité de la matière organique diminuait rapidement, passant de 82 à 65 p. Ioo environ. Il n'existe pas à notre connaissance d'étude publiée sur l'évolution de la digestibilité in vivo des céréales après la floraison. Une technique récente consistant à faucher et à ensiler la plante de céréale à partir de la maturité laiteuse du grain ou plus tard (cf. DEMarguILLY et al., I969), nous avons mesuré l'évolution de la digestibilité et de la quantité ingérée de la plante entière de céréale durant la phase de maturation du grain.

\section{MATÉRIEL E'T MÉTHODES}

La digestibilité et la quantité ingérée de la plante entière de 4 céréales sur pied ont été mesurées sur des moutons entre le stade "début épiaison " ou "floraison " et la fin du stade "pâteux ", soit, durant 7 à 9 semaines consécutives. Ces 4 céréales, une avoine Condor en I968, un blé Artois en 1968, un blé Cappelle en 1969 et une orge Iris en 1967 avaient été semées dans des parcelles du Centre de Recherches zootechniques et vétérinaires de Theix (80o m d'altitude) dans le Puy-de-Dôme, l'avoine et l'orge avaient été semées à la dose de $120 \mathrm{~kg} / \mathrm{ha}$ et le blé à I $60 \mathrm{~kg} / \mathrm{ha}$, mais le nombre de plantes au $\mathrm{m}^{2}$ n'a pas été mesuré. Toutes ces céréales avaient reçu une fumure de $90 \mathrm{~kg}$ de $\mathrm{P}$ et $\mathrm{K}$ par hectare lors du labour et de roo $\mathrm{kg}$ de $\mathrm{N}$ à la fin avril.

Chaque céréale fauchée le matin, puis hachée en brins de 3 à $4 \mathrm{~cm}$ de longueur, a été distribuée à volonté (5 à ro p. Ioo de refus) chaque jour en 3 repas ( $8 \mathrm{~h} 30$, I $3 \mathrm{~h} 30$, I $7 \mathrm{~h}$ ) à un lot de 4 moutons en cage à métabolisme et de 2 moutons en case individuelle. La digestibilité et la quantité ingérée ont été mesurées en continu; chaque période de mesures allant ḑu lundi au samedi, était séparée de la suivante par un seul jour, le dimanche.

Les surfaces et les quantités fauchées ont été mesurées chaque jour. Au milieu de chaque période, un échantillon de $500 \mathrm{~g}$ a été prélevé et les plantes séparées en épis, tiges + gaines et limbes. Ces différentes fractions ont été séchées à $80^{\circ} \mathrm{C}$ dans une étuve ventilée puis pesées et leur poids a été exprimé en $\mathrm{p}$. Ioo du poids de la matière sèche totale.

Les échantillons représentatifs de la céréale offerte, de la céréale refusée et des fèces, correspondant à chaque période ont été analysés pour déterminer leur teneur en cendres, en matières azotées et en cellulose brute Weende. Nous avons en outre déterminé la teneur en glucides solubles par la méthode de Somogyi (1952) et en amidon par la méthode de Thivend et al. (r965) des échantillons représentatifs des céréales offertes.

\section{RÉSULTATS ETT DISCUSSION}

\section{Composition morphologique et chimique}

La participation relative des divers organes à la matière sèche de la plante évolue régulièrement jusqu'au stade pâteux (fig. I). La part de l'épi dans la plante a augmenté de façon importante; elle est passée à la floraison de I2-I5 p. Ioo chez le blé et l'orge et de I8-20 p. Ioo chez l'avoine à 50-55 p. Ioo environ au stade pâteux quelle que soit l'espèce. En revanche, la proportion de tiges + gaines a diminué de 6o-65 p. Ioo à 40-45 p. Ioo, et celle de limbes de 20-25 p. Ioo à 7-Io p. Ioo. Les résultats confirment ceux obtenus lors d'une étude beaucoup plus complète effectuée dans le cadre d'une action concertée intra I. N. R. A. sur les céréales immatures, sur de nombreuses variétés de blé et d'orge cultivées au Centre de Recherches agronomiques de Clermont-Ferrand (DEmarquiliy et al., ig69).

La teneur en matière sèche de la plante a augmenté régulièrement de I5-20 p. Ioo à la floraison à 35-40 p. Ioo au stade pâteux. Dans le même temps, la teneur en 
cendres a diminué de Io p. Ioo à 7 p. Ioo environ, celle en cellulose brute de 33 à 27-28 p. Ioo et celle en matières azotées de ro à $7 \mathrm{p}$. roo chez le blé et l'avoine et de I2 à 9 p. Ioo chez l'orge (tabl. I).
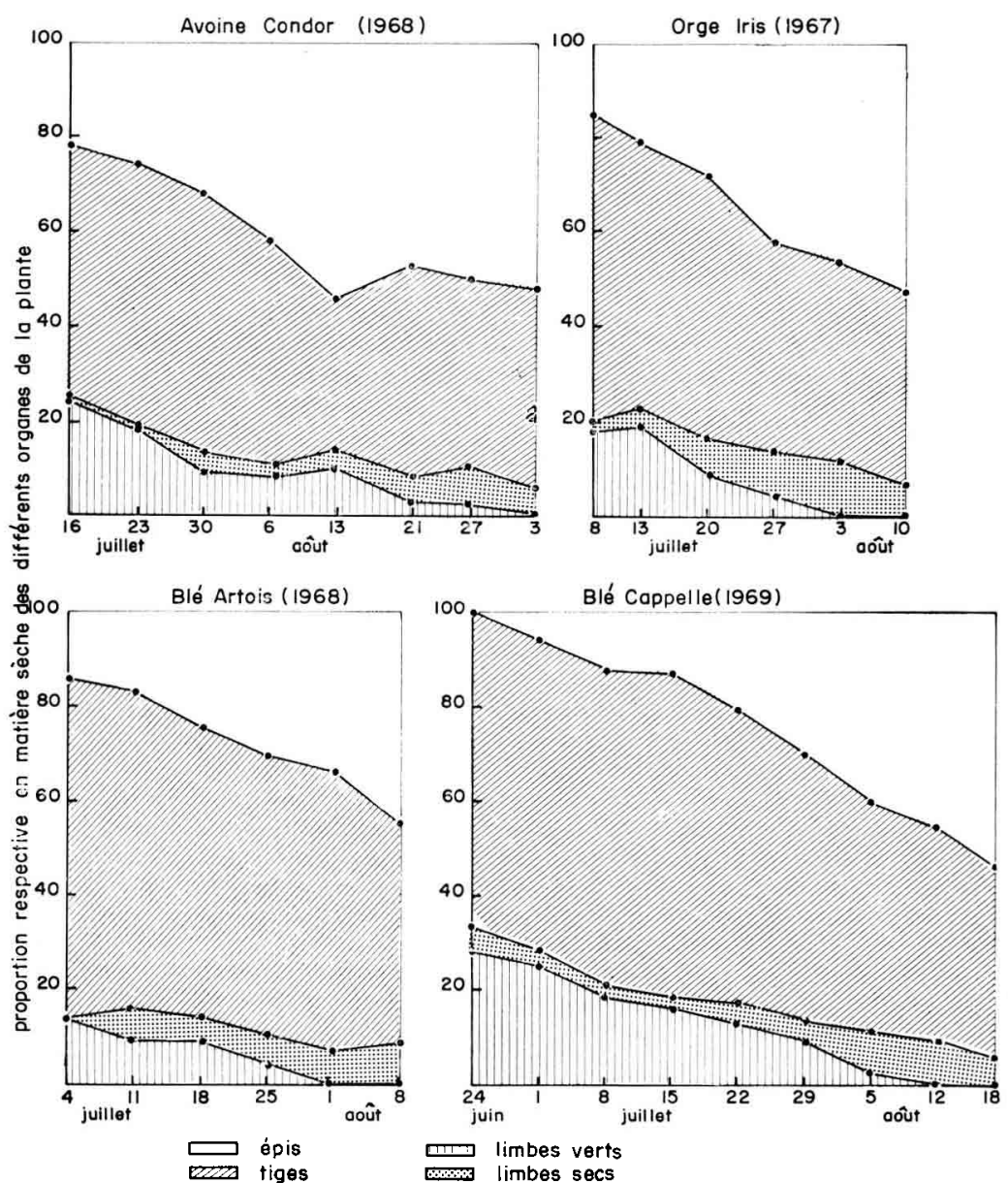

FIG. I. - Evolution au cours de la maturité de la composition morphologique de la plante de diverses céréales

Les teneurs en constituants solubles dans l'eau et en glucides solubles de la plante évoluent parallèlement; elles augmentent jusqu'au début du stade laiteux et diminuent ensuite très rapidement (tabl. I). En particulier la teneur en glucides solubles qui est en moyenne de 7,5 p. Ioo à la floraison, passe à I2,9 p. I00 au début du stade laiteux pour diminuer à $4,7 \mathrm{p}$. Ioo au stade pâteux. La diminution de la teneur en glucides solubles doit résulter essentiellement de leur transformation en amidon puisque dans le même temps, la teneur en amidon de la plante passe de 3,5 à I9,o p. Ioo en moyenne. 


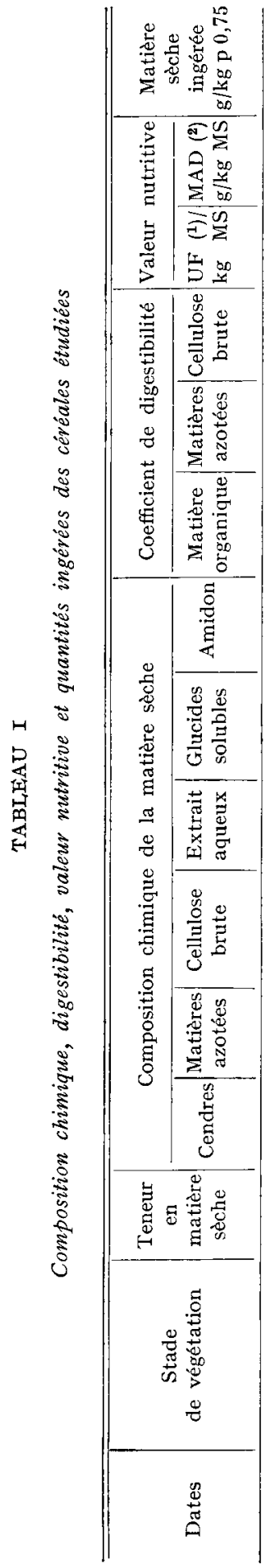

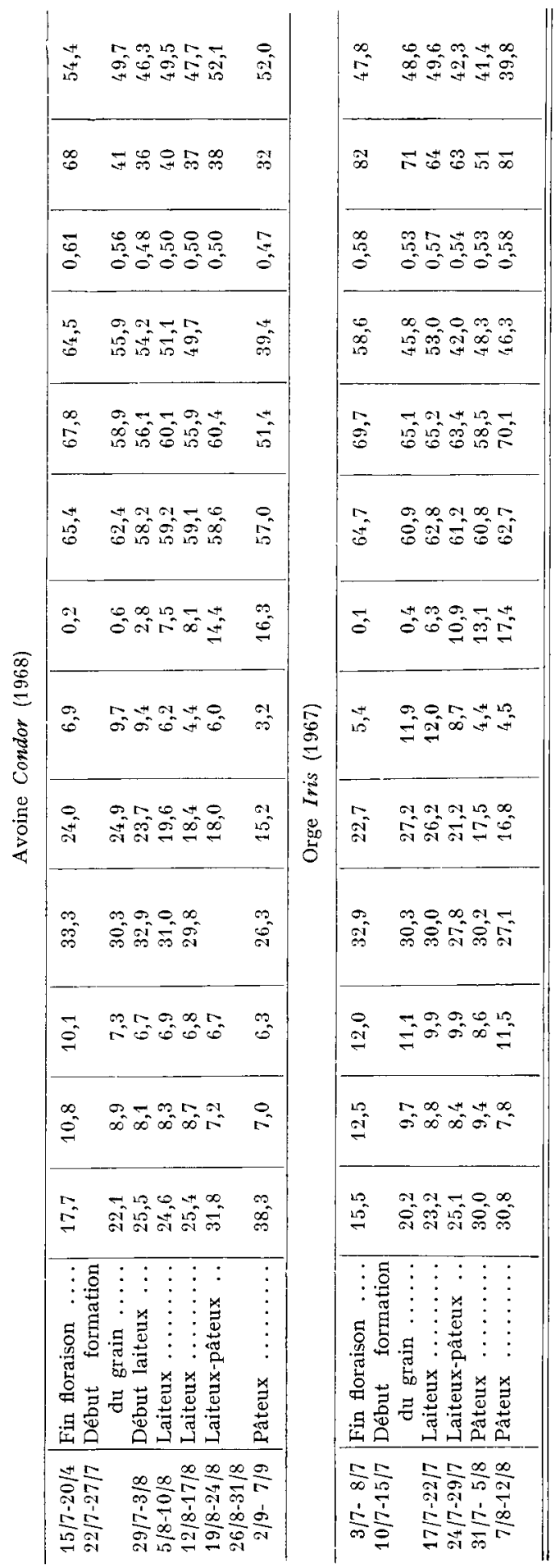




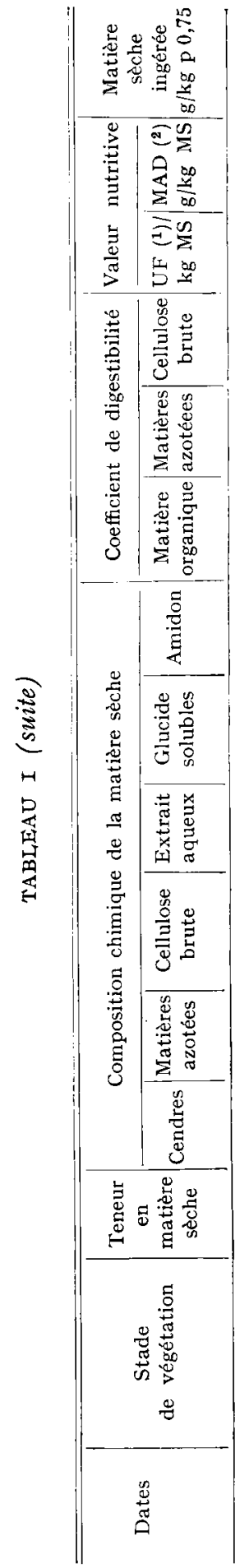

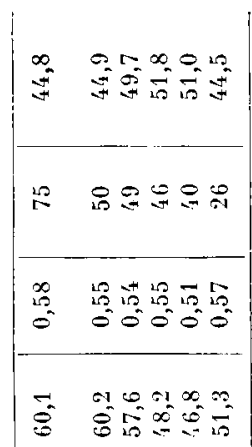

0
0
0

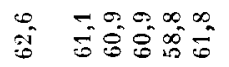

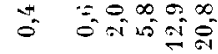

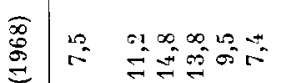

$\frac{1}{5}$

ए ค

m की

$\stackrel{0}{0} \quad \infty \begin{aligned} & 0 \\ & 0\end{aligned}$

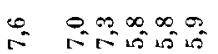

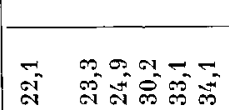

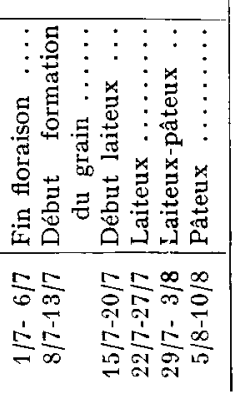

\begin{tabular}{|c|c|}
\hline $\begin{array}{ll}6 \\
0 \\
0 \\
0\end{array}$ & 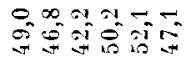 \\
\hline 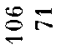 & 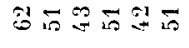 \\
\hline $\begin{array}{l}0 \\
0.98 \\
0\end{array}$ & 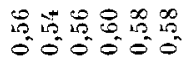 \\
\hline $\begin{array}{l}\theta_{n}=0 \\
\vec{\sigma}=\dot{E}\end{array}$ & 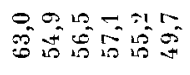 \\
\hline
\end{tabular}

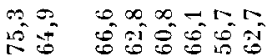

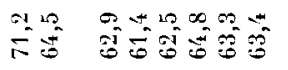

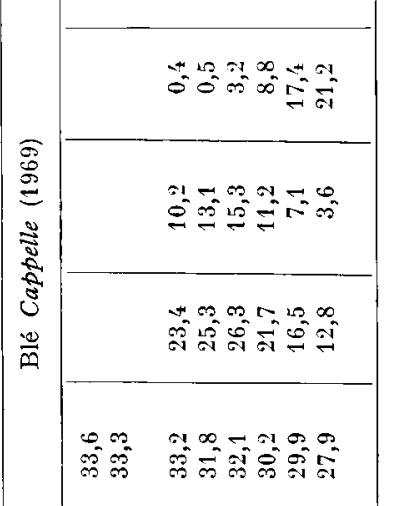

等

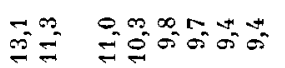

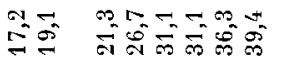

\begin{tabular}{|c|c|}
\hline 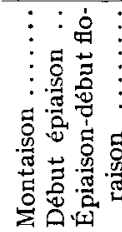 & 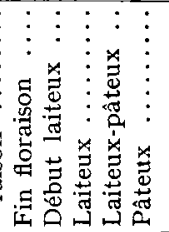 \\
\hline 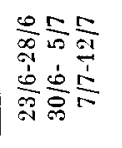 & 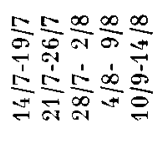 \\
\hline
\end{tabular}

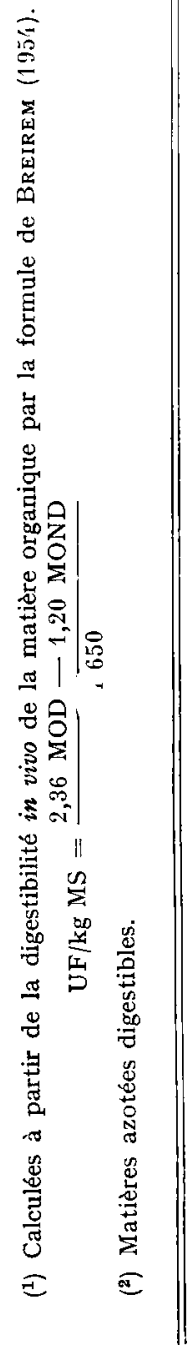




\section{Digestibilité}

$L_{1}$ a digestibilité de la matière organique a diminué rapidement jusqu'au début de la formation du grain où elle était de l'ordre de $6 \mathrm{I}-62 \mathrm{p}$. Ioo chez les 4 céréales. Elle est ensuite restée pratiquement constante jusqu'au stade pâteux chez l'orge et le blé Artois. En revanche, elle a augmenté jusqu'au stade laiteux chez le blé Cappelle et est restée alors comprise entre 63 et 64 p. Ioo tandis qu'elle a continué à décroître lentement chez l'avoine atteignant 59 p. Ioo au stade laiteux et 57 p. Ioo au stade pâteux (fig. 2).
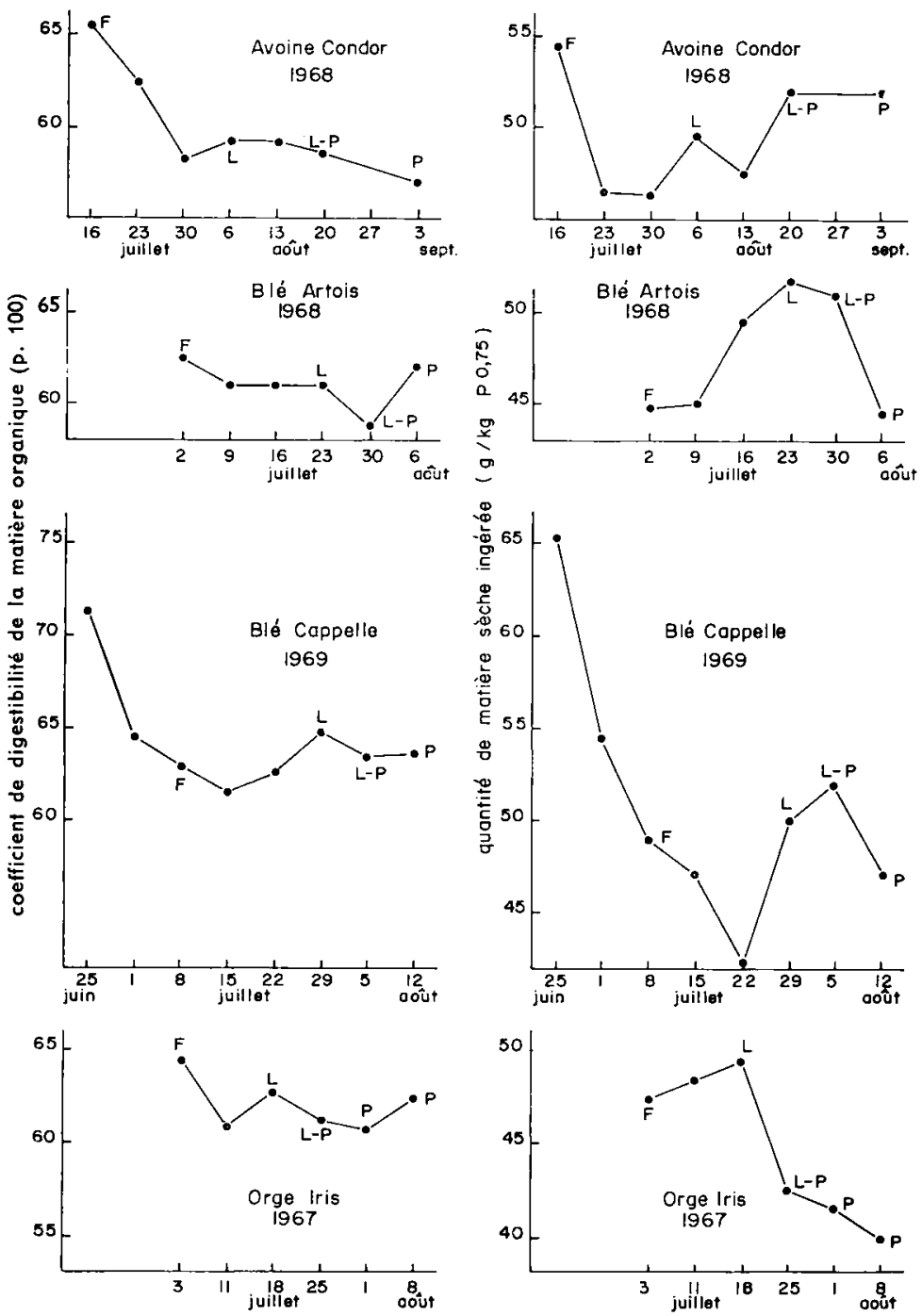

FIG. 2. - Évolution de la digestibilité et de la quantité ingérée de 4 céréales plante entière au cours de la phase de maturation du grain

(F : Floraison; L : stade laiteux ; L. P. stade laiteux-pâteux ; P : stade pâteux) 
La digestibilité de la matière organique de la plante entière de blé et d'orge restant pratiquement constante à partir soit de la floraison, soit du stade laiteux, alors que la composition morphologique et chimique continue à évoluer, il n'est pas étonnant qu'elle ne soit que très faiblement liée aux divers critères morphologiques ou chimiques de la plante et notamment à la teneur en cellulose brute $\left(r=+0,395^{*}\right.$, $n=26)$ ou en matières azotées $\left(r=0,733^{* *}\right.$ pour l'ensemble des 27 échantillons et $r=0,623$ si on supprime l'échantillon de blé étudié au stade montaison) (fig. 3).

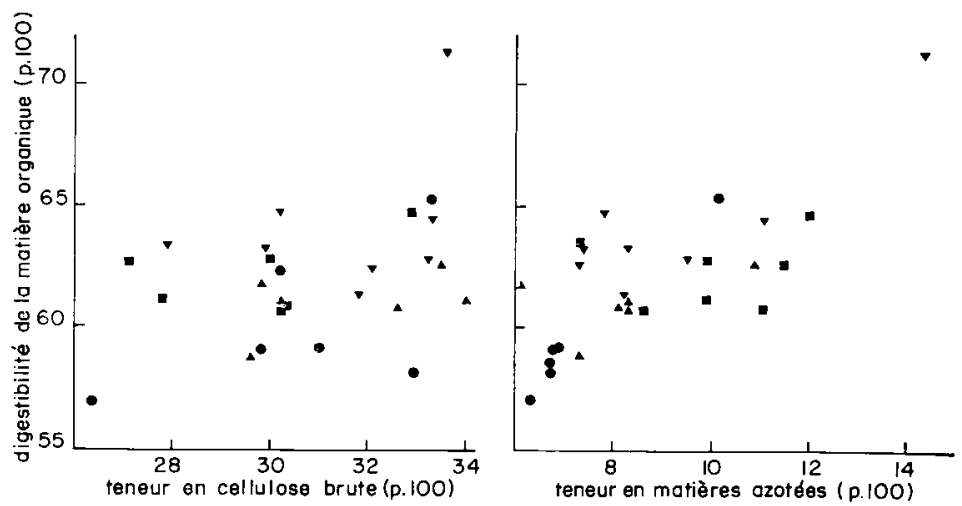

Fig. 3. - Relation entre la digestibilité de la matière organique des plantes d'avoine (•)

d'orge (a) et de blé (৯) et la teneur en cellulose brute ou la teneur en matières azotées des plantes

Les coefficients de corrélation calculés par espèce ne sont pas significatifs à l'exception de celui liant la digestibilité de la matière organique et la teneur en matières azotées des 7 échantillons d'avoine : $r=0,927$. Cette constance de la digestibilité a été retrouvée in vitro chez diverses variétés d'orge par EDwards et al. (I968) et chez diverses variétés de blé et d'orge par nous-mêmes (DEMarqurLLy et al., I 969 ) et par LENOBLE (résultats non publiés) de la Station d'Amélioration des Plantes de Lusignan. Elle résulte du fait que l'augmentation dans la plante entière du grain dont la digestibilité est élevée et constante, compense la diminution de digestibilité de tous les autres organes de la plante et notamment de la tige.

La digestibilité de la cellulose brute a diminué en même temps que la teneur. De l'ordre de $65 \mathrm{p}$. Ioo à la floraison, elle était au stade pâteux de $5^{\circ} \mathrm{p}$. Ioo pour le blé, $45 \mathrm{p}$. Ioo pour l'orge et $40 \mathrm{p}$. Ioo pour l'avoine.

La digestibilité des matières azotées a varié parallèlement à la teneur en matières azotées de chaque espèce. Il existe cependant des différences systématiques entre espèces puisque les teneurs en matières azotées non digestibles ont été respectivement pour l'avoine, le blé et l'orge de 3 , I $\pm 0, I_{5} ; 3,3 \pm 0, I_{4}$ et $3,6 \pm 0,24$.

\section{Quantité ingérée}

La quantité de matière sèche ingérée a diminué rapidement jusqu'au début de la formation du grain où elle a été de 1'ordre de $45 \mathrm{~g} / \mathrm{kg} \mathrm{P}^{0,75}$ quelle que soit l'espèce. Pour le blé et 1'orge elle a alors augmenté légèrement de Io à I 5 p. Ioo jusqu'au stade laiteux pour diminuer rapidement ensuite, notamment chez l'orge. L'augmentation de la quantité d'avoine ingérée a été du même ordre que pour les blés mais elle a été plus lente, le maximum étant atteint au stade pâteux (fig. 2). Il n'existe 
pas de liaison significative entre la quantité de matière sèche ingérée et soit le coefficient de digestibilité de la matière organique, soit les différents critères morphologiques ou chimiques de la plante.

\section{Production à l'hectare}

La quantité de matière sèche récoltée à l'hectare a augmenté rapidement jusqu'au stade laiteux, puis lentement jusqu'au début du stade pâteux où elle a atteint son maximum qui a été de l'ordre de 10,6 tonnes pour l'avoine, I3,0 tonnes pour les blés et 8,5 tonnes pour l'orge dont le peuplement était insuffisant (tabl. 2). La digestibilité de la matière organique et par là vraisemblablement la valeur énergétique restant pratiquement constante dès le stade laiteux (de l'ordre de $0,50 \mathrm{UF} / \mathrm{kg}$ de matière sèche pour l'avoine et comprise entre 0,55 et 0,60 UF pour les blés et l'orge), la quantité d'UF récoltée à 1'hectare a atteint son maximum (5 300 UF pour l'avoine, 7500 UF pour les blés et 4500 UF pour 1'orge) en même temps que la quantité de matière sèche, soit au début du stade pâteux. C'est donc à ce stade qu'il faudra exploiter les céréales pour les ensiler, d'autant plus que leur teneur en matière sèche (comprise entre 35 et $40 \mathrm{p}$. Ioo) est alors optimum pour la réussite

\section{TABLEAU 2}

Quantité de matière sèche et d'UF récoltées à l'hectare sous forme de céréales immatures et sous forme de grain et de paille à maturité

\begin{tabular}{|c|c|c|c|c|c|c|c|c|}
\hline & \multirow[b]{3}{*}{ Année } & \multirow[b]{3}{*}{$\begin{array}{l}\text { Stade } \\
\text { de maturité }\end{array}$} & \multirow{2}{*}{\multicolumn{2}{|c|}{$\begin{array}{c}\text { Quantité récoltée } \\
\text { au stade immature } \\
\text { (fauche à } 10 \mathrm{~cm} \\
\text { du sol) }\end{array}$}} & \multicolumn{4}{|c|}{ Quantité récoltée à maturité } \\
\hline & & & & & \multirow[b]{2}{*}{$\begin{array}{l}\text { Matière } \\
\text { sèche } \\
\text { totale }\end{array}$} & \multicolumn{2}{|c|}{ Grain } & \multirow[b]{2}{*}{$\begin{array}{c}\text { Paille } \\
\text { (tonne) }\end{array}$} \\
\hline & & & $\begin{array}{l}\text { Matière } \\
\text { sèche } \\
\text { (tonne) }\end{array}$ & U.F.* & & $\begin{array}{c}\text { Matière } \\
\text { sèche } \\
\text { (tonne) }\end{array}$ & U.F.** & \\
\hline Avoine Condor & 1968 & $\begin{array}{l}\text { Floraison } \ldots \ldots \ldots \\
\text { Laiteux } \ldots \ldots \ldots \ldots \\
\text { Laiteux-pâteux } \ldots \\
\text { Pâteux } \ldots \ldots \ldots\end{array}$ & $\begin{array}{r}5,4 \\
9,7 \\
10,6 \\
10,6\end{array}$ & $\begin{array}{l}3300 \\
4850 \\
5300 \\
5000\end{array}$ & 11,3 & 3,52 & 3170 & 7,8 \\
\hline Blé Artois & 1968 & $\begin{array}{l}\text { Floraison } . . . \cdots \\
\text { Laiteux } \ldots \ldots \ldots \\
\text { Laiteux-pâteux } \ldots \\
\text { Pâteux }\end{array}$ & $\begin{array}{r}9,5 \\
12,1 \\
12,8\end{array}$ & $\begin{array}{l}5530 \\
6650 \\
6530\end{array}$ & 13,0 & $3,6 / 4$ & 1370 & 9,4 \\
\hline Blé Cappelle & 1969 & $\begin{array}{l}\text { Floraison } \ldots \ldots \ldots \\
\text { Laiteux } \ldots \ldots \ldots \\
\text { Laiteux-pâteux } \ldots \\
\text { Pâteux } \ldots \ldots \ldots\end{array}$ & $\begin{array}{r}9,9 \\
12,2 \\
12,6 \\
13,3\end{array}$ & $\begin{array}{ll}5 & 540 \\
7 & 320 \\
7 & 350 \\
7 & 700\end{array}$ & 13,7 & 4,86 & 5830 & 8,9 \\
\hline Orge Iris & 1967 & $\begin{array}{l}\text { Floraison } \ldots \ldots \ldots \\
\text { Laiteux } \ldots \ldots \ldots \\
\text { Laiteux-pâteux } \ldots \\
\text { Pâteux } \ldots \ldots \ldots\end{array}$ & $\begin{array}{l}6,1 \\
7,9 \\
8,0 \\
8,5\end{array}$ & $\begin{array}{l}3500 \\
4500 \\
4300 \\
4500\end{array}$ & & $\begin{array}{c}\text { non } \\
\text { mesurée }\end{array}$ & & \\
\hline
\end{tabular}

* Calculées à partir de la digestibilité de la matière organique in vivo par la formule de Breirem (1954).

** Calculées à partir des valeurs fourragères des grains données dans les tables de Leror. 
de l'ensilage (DEMARQUILLY et al., I969). Ía quantité de matières azotées digestibles récoltée à l'hectare a, quant à elle, atteint son maximum beaucoup plus tôt, soit entre la floraison et le début du stade laiteux suivant les espèces.

Pour l'avoine et les deux blés nous avons pu estimer les quantités de matière sèche de grain et de paille obtenues à maturité complète à partir de 4 échantillons témıins de $4 \mathrm{~m}^{2}$ chacun. Celles-ci sont présentées dans le tableau 2 en parallèle avec celles qui ont été récoltées sous forme de plante entière immature. Si les quantités de matière sèche totale récoltées au stade immature et à maturité sont peu différentes, la quantité d'UF obtenue sous forme de plante entière immature est supérieure de I 870 à 28 Io UF à celle récoltée sous forme de grain sec seul. Cependant cette comparaison n'est peut-être pas valable car la valeur énergétique (exprimée en unités fourragères) n'a pas été obtenute avec le même mode de calcul (voir notes du tab1. 2).

En conclusion, ces résultats montrent que la plante de céréales (de blé notamment) exploitée au stade laiteux-pâteux ou début pâteux peut être compétitive vis-à-vis des fourrages classiques pour fournir l'élément principal de la ration de base hivernale des ruminants, tout au moins dans les zones où les céréales viennent régulièrement et où le maîs ne peut croître de façon satisfaisante ou atteindre une maturité suffisante pour être ensilé dans de bonnes conditions (à 30 p. Ioo de matière sèche). Un des intérêts des céréales ensilées réside dans la possibilité que l'on a, en faisant varier la hauteur de coupe lors de la récolte, de modifier la concentration énergétique de l'ensilage suivant les besoins des animaux auxquels l'ensilage est destiné. Pour les variétés de taille normale, la digestibilité augmente en effet d'environ Io points quand la hauteur de coupe s'élève de $10 \mathrm{~cm}$ du sol à $15 \mathrm{~cm}$ sous l'épi (Demarquiliy et al., ig69; ANDrieu et Demarquil, iy, r969).

Reçu pour publication en octobre 1970.

\title{
SUMMARY
}

\author{
CHANGE IN THE DIGESTIBILITY AND DRY MATTER INTAKE \\ OF WHOLE PLAN'TS OF OAT, WHEAT AND BARLEY \\ STUDIED BETWEEN FLOWERING AND GRAIN RIPENNING
}

The digestibility and dry matter intake of whole oat, barley and wheat crops were measured in sheeps from the early ear emergence stage or flowering and until the end of the dough stage.

The relative amount of dry matter in the different organs of the plants changes regularly until the dough stage (fig. I). During ripening, the crude protein and crude fiber content decreases, whereas the amount of starch increases (table I).

Until the beginning of development of the grain, the digestibility of the organic matter rapidly decreases down to $6 \mathrm{I}-62 \mathrm{p}$. Ioo in the three cereals; it then either remains almost constant until the dough stage in the case of barley and wheat or increases again a little until the milk stage and then stabilizes. In the case of oat, this digestibility goes on decreasing very slowly (fig. 2, table I). It is slightly correlated with the crude protein content $(r=0,733, n=27)$ and even less with the crude fiber content $(r=0,395, n=26)$ (fig. 3).

The dry matter intake decreases rapidly down to $45 \mathrm{~g} / \mathrm{kg} \mathrm{P}^{0,75}$ at the beginning of development of the grain. From that time there is a slight increase from Io-I 5 p. roo until the milk stage for wheat and barley and until the dough stage in the case of oat, whereafter a rapid decrease takes place (fig. I). 
The dry matter harvest per hectare is high and increases rapidly from the moment of flowering attaining a maximum at the very beginning of the dough stage (table 2).

The milk-dough stage constitutes the optimum period of harvesting the cereals for silage when the dry matter content is between 35 and $40 \mathrm{p}$. roo.

\section{RÉFÉRENCE,S BIBI,IOGRAPHIQUES}

Andrieu J., Demarquilly C., r969. Valeur alimentaire pour le ruminant des céréales immatures. Entreprise agricole, octobre I969. Étude 1602 .

Demarquiliy C., Paquet J., Andriev J., ig69. Les céréales immatures. Bull. tech. Inf. Ingrs Servs agric., 244, 885-904.

Edwards Ra., Donalson Elizabeth, Mac Gregor A. W., I968. Ensilage of whole crop barley. I. Effects of variety and stage of growth. J. Sci. Fd. Agric., 19,655-66o,

NeHRING K., BeYer M., I 965 . ¿ Cber zusammensetzung und futterwert von grün und rauhfutterstoffen. I. Mitteilung. Der futterwert von grünroggen in Abhängigkeit von schnittzeit, N-Düngung und Sorte. Arch. Tievernähr, 15, 283-308.

NEHRING K., BEYER M., I966. Ujber zusammensetzung und futterwert von grün und rauhfutterstoffen. 2. Mitteilung. Der futterwert von grünhafer in Abhängigkeit von schnittzeit und N-Düngung. Arch. Tierernähr., 16, I03-124.

Somogy M., 1952. Notes on sugar determination. J. Biol Chen., 195, 19-23.

Thivend P., Mercier C., Guilbot A., i 965 . Dosage de l'amidon dans les milieux complexes. Ann. Biol. anim. Bioch. Biophys., 5, 513-526. 